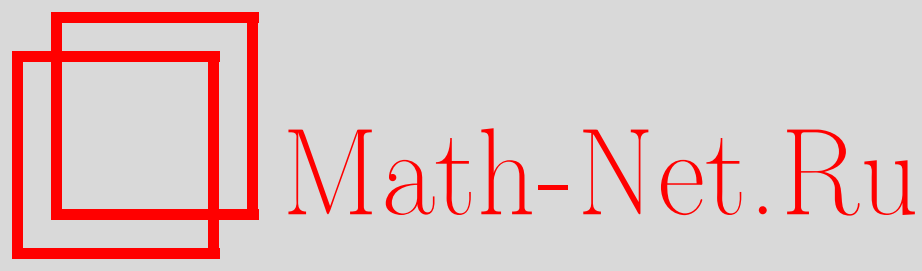

А. В. Забродин, Преобразования Беклунда для разностного уравнения Хироты и суперсимметричный анзац Бете, TMФ, 2008, том 155, номер 1, 74-93

DOI: https://doi.org/10.4213/tmf6194

Использование Общероссийского математического портала Math-Net.Ru подразумевает, что вы прочитали и согласны с пользовательским соглашением http://www.mathnet.ru/rus/agreement

Параметры загрузки:

IP : 52.87 .193 .239

26 апреля 2023 г., 14:32:51

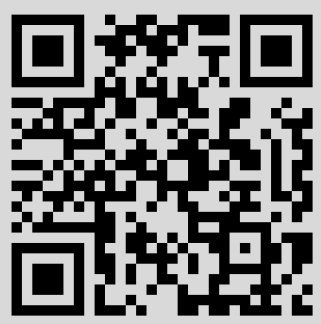




\title{
ПРЕОБРАЗОВАНИЯ БЕКЛУНДА ДЛЯ РАЗНОСТНОГО УРАВНЕНИЯ ХИРОТЫ И СУПЕРСИММЕТРИЧНЫЙ АНЗАЦ БЕТЕ
}

\begin{abstract}
Рассмотрены $G L(K \mid M)$-инвариантные интегрируемые суперсимметричные спиновые цепочки с твистованными граничными условиями и продемонстрирована роль преобразований Беклунда в решении разностного уравнения Хироты для собственных значений трансфер-матриц этих цепочек. Показано, что техника вложенных анзацев Бете эквивалентна цепочке последовательных преобразований Беклунда, "раздевающих" исходную задачу до тривиальной.
\end{abstract}

Ключевые слова: интегрируемые нелинейные разностные уравнения, преобразования Беклунда, интегрируемые суперсимметричные спиновые цепочки, анзац Бете.

\section{1. ВВЕДЕНИЕ}

В интегрируемых моделях взаимосвязь классических и квантовых систем ни в коей мере не исчерпывается их соответствием в классическом пределе. Как теперь хорошо известно, классические интегрируемые уравнения часто возникают в квантовых интегрируемых задачах даже при $\hbar \neq 0$.

Один важный пример этого общего явления был исследован в работах [1]-[3], где было показано, что спектр коммутирующих трансфер-матриц (интегралов движения) в квантовых интегрируемых моделях может быть найден в терминах также интегрируемой дискретной классической динамики, заданной в пространстве, точки которого нумеруют коммутирующие трансфер-матрицы. В случае $G L(K)$-инвариантных спиновых цепочек координатами в этом пространстве являются параметры, характеризующие конечномерные неприводимые представления группы $G L(K)$, и спектральный параметр. Классическая динамика в этом пространстве задается функциональными соотношениями для трансфер-матриц, установленными в [4]-[6] для обычного бозонного случая и распространенными на суперсимметричный случай

* Институт биохимической физики им. Н. М. Эммануэля РАН; Институт теоретической и экспериментальной физики, Москва, Россия. E-mail: zabrodin@itep.ru 
в [7]. Наиболее важным среди этих соотношений является билинейное функциональное уравнение для собственных значений трансфер-матриц ( $T$-функций), которое имеет вид билинейного разностного уравнения Хироты [8]. Для краткости мы будем называть его TT-соотношением. Оно является отправной точкой нашего подхода.

Уравнение Хироты - это, возможно, самое знаменитое уравнение в теории классических интегрируемых систем на решетке. Оно дает универсальную интегрируемую дискретизацию различных солитонных уравнений и в то же время является порождающим уравнением для их иерархий. Оно участвует в большом количестве интегрируемых задач, классических и квантовых. Как и другие солитонные уравнения, уравнение Хироты допускает (авто)преобразования Беклунда, т.е. преобразования, которые переводят любое решение в решение того же уравнения. Они позволяют построить семейство решений, связанных с некоторым особенно простым решением конечной цепочкой таких преобразований.

Преобразования Беклунда играют центральную роль в нашем методе и служат альтернативой стандартной технике анзаца Бете. Решение $G L(K)$-инвариантных спиновых цепочек с помощью вложенных анзацев Бете заключается в последовательном уменьшении ранга группы путем повторного применения анзаца Бете. Таким образом можно спускаться от $G L(K)$ к $G L(K-1)$, пока задача не станет тривиальной при $K=0$. На промежуточных стадиях этого процесса вводятся несколько вспомогательных “ $Q$-функций” (собственных значений $Q$-операторов Бакстера), связанных с $T$-функциями $T Q$-соотношениями Бакстера. Если рассматривать $Q$-функции как функции спектрального параметра, то их нули удовлетворяют системе уравнений Бете. Эта чисто квантовая техника имеет замечательную классическую интерпретацию [1], [2]: она эквивалентна цепочке преобразований Беклунда для уравнения Хироты. При этом $T Q$-соотношения появляются как составная часть вспомогательных линейных задач для уравнения Хироты. Ранг группы становится дополнительной дискретной переменной, причем зависимость от этой переменной опять описывается уравнением, подобным уравнению Хироты. Поскольку решения являются полиномами по спектральному параметру, их нули удовлетворяют уравнениям движения некоторой конечномерной динамической системы в дискретном времени. Эти уравнения движения и являются уравнениями Бете.

Недавно в работе [9] этот подход был применен к суперсимметричным спиновым цепочкам, построенным с помощью $G L(K \mid M)$-инвариантных решений градуированного уравнения Янга-Бакстера [10], [11]. В этом случае имеются два (а не один) дополнительных дискретных потока, соответствующих бозонному и фермионному рангам $K$ и $M$. Условие совместности этих потоков приводит к нетривиальному билинейному соотношению для собственных значений $Q$-операторов Бакстера $(Q Q$-соотношению). В настоящей работе мы обобщаем эти результаты на $G L(K \mid M)$-инвариантные спиновые цепочки с твистованными (квазипериодическими) граничными условиями. Параметры твистования входят в решение как непрерывные параметры преобразований Беклунда.

Мы предполагаем, что читатель знаком со стандартными понятиями и фактами теории супергрупп и их представлений [12]-[14], а также с основами квантового метода обратной задачи рассеяния [15]-[17]. 


\section{2. ТT-СООТНОШЕНИЕ}

Напомним конструкцию семейства коммутирующих трансфер-матриц в интегрируемых спиновых цепочках. Ее основные черты одинаковы для обычных и суперсимметричных моделей. Мы рассматриваем модели на решетке (спиновые цепочки) с супергруппой симметрии $G L(K \mid M)$, построенные с помощью $G L(K \mid M)$-инвариантных $R$-матриц. Такие $R$-матрицы зависят от спектрального параметра $u \in \mathbb{C}$ и действуют в тензорном произведении $V_{0} \otimes V_{1}$ двух линейных пространств, в которых заданы неприводимые представления $\pi_{0}$ и $\pi_{1}$ супергруппы $G L(K \mid M)$. Пространство $V_{0}$ обычно называется вспомогательным, а $V_{1}$ - (локальным) квантовым пространством. Мы рассмотрим случай, когда $\pi_{1}$ - векторное представление, т.е. $V_{1}=V=\mathbb{C}^{K} \oplus \mathbb{C}^{M}$, а $\pi_{0}$ - произвольное тензорное представление супергруппы. $R$-матрица дается формулой

$$
R_{01}(u)=u+2 \sum_{\alpha \beta}(-1)^{p(\beta)} \pi_{0}\left(E_{\alpha \beta}\right) \otimes e_{\beta \alpha} .
$$

Здесь $p(\beta)$ - четность индекса $\beta(p(\beta)=0$ или $p(\beta)=1), e_{\alpha \beta}$ - матрицы с элементами $\left(e_{\alpha \beta}\right)_{\alpha^{\prime} \beta^{\prime}}=\delta_{\alpha \alpha^{\prime}} \delta_{\beta \beta^{\prime}}$ и $\pi_{0}\left(E_{\alpha \beta}\right)$ - генераторы супералгебры $g l(K \mid M)$ в представлении $\pi_{0}$. Первое (скалярное) слагаемое нужно понимать как произведение $u$ и единичной матрицы $\pi_{0}(I) \otimes I_{V_{1}}$, где $I \in G L(K \mid M)$ - единичный элемент группы, а $I_{V_{1}}$ - единичный оператор в пространстве $V_{1}$. Эта $R$-матрица является $G L(K \mid M)$-инвариантным решением градуированного уравнения Янга-Бакстера [10], [11]. Инвариантность относительно супергруппы означает, что

$$
\pi_{0}(g) \otimes \pi_{1}(g) R_{01}(u)=R_{01}(u) \pi_{0}(g) \otimes \pi_{1}(g)
$$

для любого $g \in G L(K \mid M)$.

Чтобы ввести обобщенные интегрируемые спиновые цепочки на $N$ узлах, возьмем $N$ копий пространства $V=V_{1}=V_{2}=\cdots=V_{N}$ (по одному для каждого узла цепочки) и соответствующие $R$-матрицы $R_{0 i}(u)$, действующие в $V_{0} \otimes V_{i}$. Гильбертово пространство состояний модели $\mathcal{H}$ является тензорным произведением локальных квантовых пространств $V_{i}$ по всем узлам цепочки: $\mathcal{H}=\bigotimes_{i=1}^{N} V_{i}$. Мы будем называть $\mathcal{H}$ квантовым пространством модели. Квантовая матрица монодромии строится как произведение $R$-матриц $R_{0 i}(u)$ в пространстве $V_{0}$ :

$$
\mathcal{T}(u)=R_{01}\left(u-\xi_{1}\right) R_{02}\left(u-\xi_{2}\right) \ldots R_{0 N}\left(u-\xi_{N}\right) .
$$

Величины $\xi_{i}$ являются внешними данными, характеризующими (неоднородную) спиновую цепочку. Суперслед квантовой матрицы монодромии, взятый в пространстве $V_{0}$, дает семейство зависящих от $u$ и $\pi_{0}$ операторов в квантовом пространстве (они называются трансфер-матрицами), которые коммутируют между собой при всех значениях этих параметров. Более общая конструкция включает твистованные (квазипериодические) граничные условия, задающиеся с помощью диагональной матрицы

$$
g=\operatorname{diag}\left(x_{1}, \ldots, x_{K}, y_{1}, \ldots, y_{M}\right) \in G L(K \mid M)
$$

(для простоты мы отождествляем элементы супергруппы $G L(K \mid M)$ с матрицами из ее векторного представления). Тогда коммутирующее семейство трансфер-матриц дается формулой

$$
T^{\left(\pi_{0}\right)}(u ; g)=\operatorname{str}_{\pi_{0}}\left(\pi_{0}(g) \mathcal{T}(u)\right)
$$


Из градуированного уравнения Янга-Бакстера с учетом $G L(K \mid M)$-инвариантности следует, что трансфер-матрицы коммутируют при различных $u$ и $\pi_{0}$ (но не при различных $g$ !): $\left[T^{\left(\pi_{0}\right)}(u ; g), T^{\left(\pi_{0}^{\prime}\right)}\left(u^{\prime} ; g\right)\right]=0$.

Далее в этой статье нас будет особенно интересовать случай "прямоугольных" представлений $\pi_{0}$, т.е. представлений, соответствующих диаграммам Юнга прямоугольной формы. Пусть дана прямоугольная диаграмма Юнга длины $s$ и высоты $a$; обозначим через $\pi_{s}^{a}$ соответствующее представление. Определим квантовые трансфер-матрицы $T(a, s, u)$ для прямоугольных представлений во вспомогательном пространстве формулой

$$
T(a, s, u)=\operatorname{str}_{\pi_{s}^{a}}\left(\pi_{s}^{a}(g) \mathcal{T}(u-s+a)\right) .
$$

Это соотношение отличается от (5) сдвигом $u$, введенным для удобства дальнейших рассуждений. Как правило, мы не будем указывать зависимость от $g$ явно. Можно формально распространить это определение на нулевые значения $a$ и $s$, что соответствует тривиальному представлению $\pi_{0}^{a}=\pi_{s}^{0}=\pi_{\varnothing}\left(\pi_{\varnothing}(g)=1\right.$ для любого $g \in G L(K \mid M))$. Принимая во внимание, что $\pi_{\varnothing}\left(E_{\alpha \beta}\right)=0$ для всех генераторов супералгебры, мы заключаем из формул (1), (3) и (6), что

$$
T(0, s, u)=\prod_{j=1}^{N}\left(u-s-\xi_{j}\right), \quad T(a, 0, u)=\prod_{j=1}^{N}\left(u+a-\xi_{j}\right)
$$

с теми же $\xi_{j}$, что и в (3). Таким образом, мы видим, что $T(0, s, u)$ и $T(a, 0, u)$ являются тождественными операторами в квантовом пространстве, умноженными на скалярные полиномиальные функции. Эти функции представляют собой фиксированные внешние данные задачи. Положим

$$
\phi(u)=\prod_{i=1}^{N}\left(u-\xi_{i}\right)
$$

тогда $T(0, s, u)=\phi(u-s), T(a, 0, u)=\phi(u+a)$.

Построенные выше трансфер-матрицы линейно независимы, но связаны нелинейными функциональными соотношениями. В частности, известно [5], [6], что трансфер-матрицы (6) для прямоугольных представлений удовлетворяют $T T$-соотношению

$$
T(a, s, u+1) T(a, s, u-1)=T(a, s+1, u) T(a, s-1, u)+T(a+1, s, u) T(a-1, s, u) .
$$

Это знаменитое билинейное разностное уравнение Хироты [8], в котором $T$ играет роль тау-функции. Поскольку все трансфер-матрицы коммутируют между собой, они могут быть одновременно диагонализованы преобразованием подобия, не зависящим от $u$, следовательно, такое же соотношение (9) справедливо для любого из их собственных значений. Имея это в виду, мы будем считать трансфер-матрицы скалярными функциями и называть их $T$-функциями. Наша стратегия заключается в том, что мы примем уравнение (9) за основное уравнение квантовой теории и попытаемся вывести из него результаты для спектра квантовых трансфер-матриц. Для этого нужно задать граничные условия и аналитические свойства решений. Граничные условия будут обсуждаться в разделе 4. Аналитические свойства по $u$ определяются типом $R$-матрицы (см. более подробное обсуждение в работе [3]). Для квантовых спиновых цепочек с полиномиальными $R$-матрицами $(1)$ все $T$-функции 
$T(a, s, u)$ являются полиномами по $u$. Таким образом, мы приходим к необходимости изучения полиномиальных решений уравнения Хироты.

Следует отметить, что общее решение уравнения Хироты с граничными $T$-функциями при $a=0$ и $s=0$, приведенными в (7), дается детерминантной формулой Бажанова-Решетихина [4]. В нашей нормировке она имеет вид

$$
T(a, s, u)=\frac{\operatorname{det}_{1 \leqslant i, j \leqslant a} T(1, s+i-j, u+a+1-i-j)}{H(u-s, a)},
$$

где функция $H(u, a)$ определена следующим образом:

$$
H(u, 0)=\frac{1}{\phi(u)}, \quad H(u, 1)=1, \quad H(u, a)=\prod_{l=1}^{a-1} \phi(u+a-2 l) \quad \text { при } \quad a \geqslant 2 .
$$

При любых $a$ ее можно выразить через гамма-функцию:

$$
H(u, a)=2^{(a-1) N} \prod_{i=1}^{N} \frac{\Gamma\left(\frac{u+a-\xi_{i}}{2}\right)}{\Gamma\left(\frac{u-a-\xi_{i}}{2}+1\right)} .
$$

Формула (10) дает решение уравнения Хироты при произвольно выбранных функциях $T(1, s, u)$. Однако это решение, вообще говоря, не удовлетворяет требуемым граничным условиям и условиям аналитичности. В частности, правая часть при $a \geqslant 2$ имеет очевидные полюсы в нулях функции $H(u-s, a)$. Их сокращение возможно, если полиномы $T(1, s, u)$ выбраны некоторым специальным образом.

В заключение этого раздела - несколько слов о нормировке решений. Легко убедиться, что преобразование

$$
T(a, s, u) \longrightarrow f_{0}(u+s+a) f_{1}(u+s-a) f_{2}(u-s+a) f_{3}(u-s-a) T(a, s, u)
$$

с произвольными функциями $f_{i}$ не меняет вид уравнения Хироты. Фиксируя эти функции тем или иным способом, можно выбрать определенную нормировку решений. В нашей нормировке все полиномы $T(a, s, u)$ имеют одну и ту же степень $N$, равную числу узлов спиновой цепочки. Сюда формально включаются специальные случаи, когда один или более нулей некоторых из этих полиномов находятся в бесконечности; тогда степень в действительности будет меньше $N$. Другие возможные способы нормировки обсуждаются в [3], [9]. В общем случае решения (6) неприводимы, т.е. $T(a, s, u)$ не делится ни на один полином вида $f_{0}(u+s+a) f_{1}(u+s-$ a) $f_{2}(u-s+a) f_{3}(u-s-a)$, где по крайней мере один из полиномов $f_{i}(u)$ имеет степень, большую нуля.

\section{3. СЛУЧАЙ $N=0$ : ХАРАКТЕРЫ СУПЕРГРУППЫ $G L(K \mid M)$}

Прежде чем двигаться дальше, поучительно рассмотреть случай $N=0$, который оказывается довольно содержательным, хотя и простым, и потому является источником полезных аналогий и мотивировок при анализе более сложных моделей. Он также возникает в пределе $u \rightarrow \infty$ в моделях с $N>0$.

При $N=0$ спиновые степени свободы отсутствуют, и $T$-функции не зависят от $u$. Как видно из определения, они совпадают с характерами элемента $g \in G L(K \mid M)$ для прямоугольных представлений: $T(a, s, u)=\chi(a, s \mid g)$. Эти характеры зависят от параметров $x_{i}, y_{j}$, входящих в матрицу $g(4)$. Мы будем предполагать, что все 
$x_{i}, y_{j}$ - различные ненулевые числа. Для краткости будем писать $\chi(a, s)$ вместо $\chi(a, s \mid g)$ в тех случаях, когда матрица $g$ фиксирована.

Введем рациональную функцию

$$
w(t)=\frac{\prod_{m=1}^{M}\left(1-y_{m} t\right)}{\prod_{k=1}^{K}\left(1-x_{k} t\right)},
$$

где $t$ - вспомогательная переменная. Как следует из формул для характеров супергрупп [13], $w(t)$ является производящей функцией характеров $\chi(1, s)$, а обратная ей функция $1 / w(t)$ является производящей функцией характеров $\chi(a, 1)$ :

$$
w(t)=\sum_{s=0}^{\infty} \chi(1, s) t^{s}, \quad \frac{1}{w(t)}=\sum_{a=0}^{\infty}(-1)^{a} \chi(a, 1) t^{a} .
$$

Все остальные характеры (супераналоги функций Шура) выражаются через $\chi(1, s)$ или $\chi(a, 1)$ с помощью детерминантных равенств Якоби-Труди:

$$
\chi(a, s)=\operatorname{det}_{1 \leqslant i, j \leqslant a} \chi(1, s+i-j)=\operatorname{det}_{1 \leqslant i, j \leqslant s} \chi(a+i-j, 1) .
$$

Их этих формул следует билинейное соотношение для характеров прямоугольных представлений:

$$
\chi^{2}(a, s)=\chi(a+1, s) \chi(a-1, s)+\chi(a, s+1) \chi(a, s-1),
$$

которое является не зависящей от $u$ версией уравнения (9). Это соотношение известно как дискретное уравнение КдФ (см., например, [18], [19]), записанное в билинейной форме. Отметим также интегральное представление для характеров:

$$
\chi(a, s)=\frac{1}{(2 \pi i)^{a} a !} \oint_{\left|t_{1}\right|=1} \cdots \oint_{\left|t_{a}\right|=1} \prod_{1 \leqslant j<k \leqslant a}\left|t_{j}-t_{k}\right|^{2} \prod_{n=1}^{a} w\left(t_{n}\right) t_{n}^{-s-1} d t_{n},
$$

где предполагается, что все особенности функции $w(t)$ лежат вне единичной окружности. Этот $a$-кратный интеграл совпадает со статистической суммой асимметричной унитарной матричной модели, выраженной через собственные значения.

Можно формально распространить определение характеров на отрицательные значения $a, s$, положив в этом случае характеры равными нулю. Поскольку $\chi(0, s)=$ $\chi(a, 0)=1$ при $a, s \geqslant 0$, такое соглашение не противоречит уравнению (16) везде за исключением точки $a=s=0$. Для того чтобы уравнение удовлетворялось на всей $(a, s)$-плоскости, надо положить либо $\chi(0, n)=1$, либо $\chi(n, 0)=1$ для любого $n \in \mathbb{Z}$, а все остальные характеры $\chi(a, s)$, у которых $a<0$ или $s<0$, считать равными нулю. Мы выберем первую возможность (она согласована с интегральным представлением (17)). Пользуясь представлением (17), нетрудно показать, что если $a \geqslant K+1$ и $s \geqslant M+1$, то $\chi(a, s)=0$. Подытожив сказанное, мы получаем, что

$$
\begin{aligned}
& \chi(a, s)=0, \text { если } \\
& a<0, \text { или } a>0 \text { и } s<0, \text { или } a>K \quad \text { и } s>M .
\end{aligned}
$$

Мы видим, что область, в которой $\chi(a, s)$ не равны нулю тождественно, имеет форму "жирного крюка", образованного объединением полуполос $\{a \geqslant 0,0 \leqslant s \leqslant M\}$, $\{s \geqslant 0,0 \leqslant a \leqslant K\}$ и горизонтального луча $\{a=0, s<0\}$, идущего из начала координат в минус бесконечность (см. рис. 1). Обозначим эту область через $\mathrm{H}(K, M)$. Мы будем называть границы $\{a=0\}$ и $\{s=0, a \geqslant 0\}$ внешними, а границы внутри верхнего правого квадранта - внутренними. 


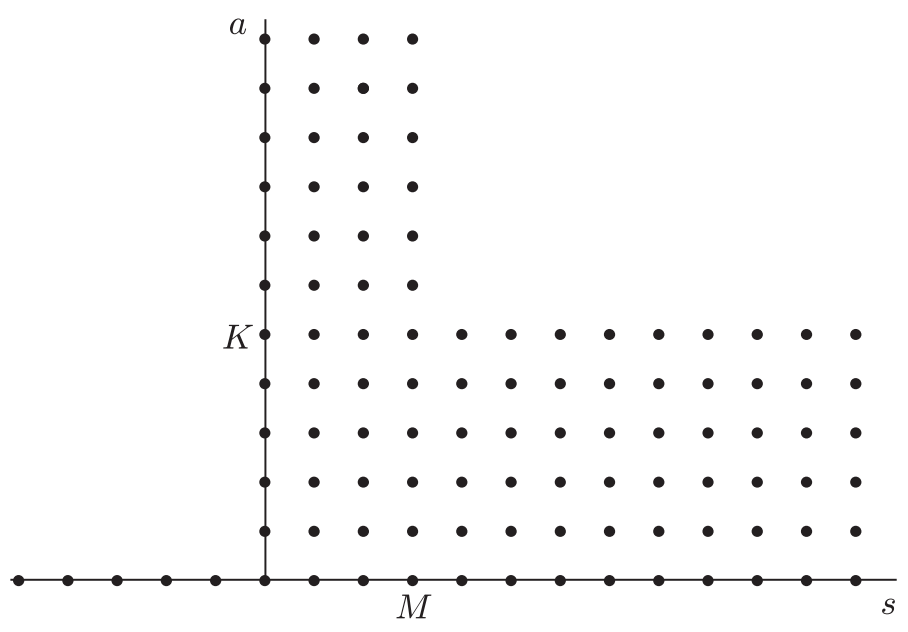

Рис. 1. Область $\mathrm{H}(K \mid M)$ на $(a, s)$-решетке при $K=5, M=3$.

Характеры на внутренних границах могут быть найдены явно. Простое вычисление показывает, что $\chi(K, M+n)$ и $\chi(K+n, M)$ при $n \geqslant 0$ записываются в виде

$$
\begin{aligned}
& \chi(K, M+n)=\left(\prod_{k=1}^{K} x_{k}^{n}\right) \prod_{i=1}^{K} \prod_{j=1}^{M}\left(x_{i}-y_{j}\right), \\
& \chi(K+n, M)=\left(\prod_{m=1}^{M}\left(-y_{m}\right)^{n}\right) \prod_{i=1}^{K} \prod_{j=1}^{M}\left(x_{i}-y_{j}\right)
\end{aligned}
$$

(на самом деле, эти формулы являются следствием более общего свойства факторизации, см. монографию [20], часть I, раздел 3, пример 23). Следовательно, они связаны соотношением

$$
\chi(K, M+n)=(-1)^{n M}(\operatorname{sdet} g)^{n} \chi(K+n, M), \quad n \geqslant 0,
$$

где

$$
\text { sdet } g=\frac{x_{1} x_{2} \ldots x_{K}}{y_{1} y_{2} \ldots y_{M}}
$$

Пользуясь детерминантными тождествами (соотношениями Плюккера) для миноров прямоугольной матрицы, составленной из элементарных характеров $\chi(1, s)$, можно доказать трехчленные билинейные соотношения

$$
\begin{aligned}
& \chi(a+1, s) \tilde{\chi}(a, s)-\chi(a, s) \tilde{\chi}(a+1, s)=z \chi(a+1, s-1) \widetilde{\chi}(a, s+1), \\
& \chi(a, s+1) \tilde{\chi}(a, s)-\chi(a, s) \tilde{\chi}(a, s+1)=z \chi(a+1, s) \tilde{\chi}(a-1, s+1),
\end{aligned}
$$

которые дают связь между характерами $\chi(a, s)$ с производящей функцией $w(t)$ и характерами $\widetilde{\chi}(a, s)$ с производящей функцией $\tilde{w}(t)=(1-z t) w(t)$. Как $\chi$, так и $\widetilde{\chi}$ удовлетворяют дискретному уравнению КдФ (16), и потому соотношения (22) задают преобразование Беклунда для него. Их смысл состоит в том, что они связывают характеры представлений $G L(k \mid m)$ с различными $k, m$. В самом деле, пусть $g_{k, m} \in G L(k \mid m)$ - диагональная матрица,

$$
g_{k, m}=\operatorname{diag}\left(x_{1}, \ldots, x_{k}, y_{1}, \ldots, y_{m}\right),
$$


полученная из $g=g_{K, M}(4)$ удалением $K-k$ собственных значений $x_{K}, x_{K-1}, \ldots$ $\ldots, x_{k+1}$ и $M-m$ собственных значений $y_{M}, y_{M-1}, \ldots, y_{m+1}$. Положим

$$
\chi_{k, m}(a, s)=\chi\left(a, s \mid g_{k, m}\right)
$$

и аналогично $(13),(14)$ введем производящую функцию характеров $\chi_{k, m}(1, s)$ :

$$
w_{k, m}(t)=\frac{\prod_{i=1}^{m}\left(1-y_{i} t\right)}{\prod_{j=1}^{k}\left(1-x_{j} t\right)} .
$$

Очевидные рекуррентные соотношения

$$
\begin{aligned}
& w_{k-1, m}(t)=\left(1-x_{k} t\right) w_{k, m}(t), \\
& w_{k, m+1}(t)=\left(1-y_{m+1} t\right) w_{k, m}(t)
\end{aligned}
$$

позволяют применить формулы $(22)$, в которых надо положить $\chi(a, s)=\chi_{k, m}(a, s)$, $\tilde{\chi}(a, s)=\chi_{k-1, m}(a, s)$ при $z=x_{k}$ или $\chi(a, s)=\chi_{k, m-1}(a, s), \tilde{\chi}(a, s)=\chi_{k, m}(a, s)$ при $z=y_{m}$.

\section{4. ГРАНИЧНЫЕ УСЛОВИЯ}

Само по себе уравнение Хироты имеет множество решений самой различной природы. Наиболее важным дополнительным требованием, позволяющим выделить класс решений, относящихся к квантовым интегрируемым моделям, являются граничные условия по переменным $a, s$. Качественно они аналогичны условиям на характеры прямоугольных представлений супергрупп, которые обсуждались в предыдущем разделе, и могут быть получены с помощью схожих аргументов. Кроме того, из явной формулы (1) для $R$-матрицы следует, что старший коэффициент полиномиальной $T$-функции совпадает с соответствующим характером: $T(a, s, u)=\chi(a, s) u^{N}+O\left(u^{N-1}\right)$ при $u \rightarrow \infty$.

Отличительной особенностью интересующих нас решений является то, что они должны тождественно обращаться в нуль в некоторых частях $(a, s)$-плоскости. Область, в которой они не равны тождественно нулю, зависит от алгебры симметрии квантовой модели. Для $G L(K \mid M)$-инвариантных суперсимметричных спиновых цепочек такой областью является $\mathrm{H}(K, M)$, введенная в предыдущем разделе (рис. 1$)$; для $G L(K)$-инвариантных спиновых цепочек она вырождается в объединение полуполосы $\{0 \leqslant a \leqslant K, s \geqslant 0\}$ с двумя лучами $\{s=0, a \geqslant K\}$ и $\{a=0, s \leqslant 0\}$. Подобно (18) мы можем написать

$$
\begin{gathered}
T(a, s, u)=0, \text { если } \\
a<0, \text { или } a>0 \text { и } s<0, \text { или } a>K \quad \text { и } \quad s>M .
\end{gathered}
$$

Легко видеть, что форма области $\mathrm{H}(K, M)$ не противоречит уравнению Хироты на всей $(a, s)$-плоскости. Хотя непосредственную физическую интерпретацию имеют только точки с неотрицательными $a, s$, важно рассматривать полную область ("жирный крюк" с лучом), поскольку иначе уравнение Хироты нарушается в угловой точке в начале координат.

Граничные значения $T$-функций имеют весьма специальную факторизованную форму, которая фиксируется условием совместности с уравнением Хироты. Действительно, на границе один из двух членов в правой части (9) становится равным 
нулю, что приводит к определенным условиям на вид граничных значений. Например, при $a=0$ уравнение (9) превращается в уравнение

$$
T(0, s, u+1) T(0, s, u-1)=T(0, s+1, u) T(0, s-1, u),
$$

которое представляет собой дискретную версию уравнения Даламбера с общим решением $T(0, s, u)=f_{+}(u+s) f_{-}(u-s)$, где $f_{ \pm}-$произвольные функции. Аналогичным образом $T(a, 0, u)=\tilde{f}_{+}(u+a) \tilde{f}_{-}(u-a)$. Уравнения $(7)$ показывают, что в нашей нормировке $f_{+}(u)=\tilde{f}_{-}(u)=1$, а $f_{-}(u)=\tilde{f}_{+}(u)=\phi(u)$, где функция $\phi(u)$ определена в (8). Аналогичная факторизация имеет место на внутренних границах. Однако обе функции в произведении, вообще говоря, нетривиальны и подлежат определению из $T T$-соотношения. Случай обычной группы $G L(K)=G L(K \mid 0)$, в котором вертикальная внутренняя граница совпадает с внешней, является в этом отношении специальным. В этом случае все граничные значения входят в задачу как фиксированные данные. В частности, на горизонтальной внутренней границе $T(K, s, u)=\phi(u+s+K)$.

На внутренних границах мы налагаем условие

$$
T(K, M+n, u)=(-1)^{n M}(\operatorname{sdet} g)^{n} T(K+n, M, u), \quad n \geqslant 0,
$$

которое согласовано с условием (21) на характеры супергруппы. В периодическом случае (когда $g$ - единичная матрица) это условие было отмечено в работе [7]. Равенство (28) означает, что значения $T$-функций в тех точках внутренней границы, которые находятся на одинаковом расстоянии от угловой точки, отличаются только постоянным множителем. Отметим, что это условие тривиально выполняется в случае $M=0$, когда граничные значения фиксированы с самого начала как данные задачи. Как уже отмечалось, при произвольных $K, M>0$ эти значения уже не фиксированы и подлежат определению вместе с $T$-функциями внутри области $\mathrm{H}(K \mid M)$.

В заключение этого раздела отметим, что параметры $x_{k}, y_{m}$ не входят явно ни в уравнение Хироты, ни в граничные условия для него. Это означает, что уравнение Хироты с фиксированными граничными условиями вида $(7)$ имеет $(K+$ $M)$-параметрическое множество полиномиальных решений.

\section{5. ВСПОМОГАТЕЛЬНЫЕ ЛИНЕЙНЫЕ ЗАДАЧИ}

Как и почти все известные нелинейные интегрируемые уравнения, уравнение Хироты является условием совместности для переопределенной системы линейных задач [1], [21]. Чтобы ввести их, удобно перейти к "киральным" переменным

$$
p=\frac{u-s-a}{2}, \quad q=\frac{u+s+a}{2}, \quad r=\frac{-u-s+a}{2} .
$$

Исходные переменные $a, s, u$ будем называть “лабораторными”. Приведем формулы для обратного преобразования,

$$
a=q+r, \quad s=-p-r, \quad u=p+q,
$$

и для преобразования векторных полей,

$$
\partial_{p}=\partial_{u}-\partial_{s}, \quad \partial_{q}=\partial_{u}+\partial_{a}, \quad \partial_{r}=\partial_{a}-\partial_{s} .
$$


Положим $\tau(p, q, r)=T(q+r,-p-r, p+q)$ и рассмотрим следующие линейные задачи для вспомогательной функции $\psi=\psi(p, q, r)$ :

$$
\begin{aligned}
& \psi(r+1)+z \frac{\tau(p+1, r+1) \tau}{\tau(p+1) \tau(r+1)} \psi=\psi(p+1), \\
& \psi(r+1)-z \frac{\tau(q+1, r+1) \tau}{\tau(q+1) \tau(r+1)} \psi=\psi(q+1),
\end{aligned}
$$

где $z$ - некоторый параметр. В этих формулах явно указаны только те переменные, которые подвергаются сдвигу. С помощью этих уравнений функция $\psi(p+1, q+1)$ может быть представлена как линейная комбинация функций $\psi(r), \psi(r+1)$ и $\psi(r+2)$ двумя разными способами. Совместность линейных задач означает, что результаты должны совпадать. Приравняв друг другу получающиеся выражения, мы видим, что члены, пропорциональные $z^{2} \psi(r)$ и $\psi(r+2)$, сокращаются непосредственно, а члены, пропорциональные $z \psi(r+1)$, дают нетривиальное соотношение (при условии, что $\psi(r+1)$ не обращается в нуль)

$$
\begin{aligned}
& \frac{\tau(p+1, r+2) \tau(r+1)}{\tau(p+1, r+1) \tau(r+2)}-\frac{\tau(p+1, q+1, r+1) \tau(p+1)}{\tau(p+1, q+1) \tau(p+1, r+1)}= \\
& \quad=\frac{\tau(p+1, q+1, r+1) \tau(q+1)}{\tau(p+1, q+1) \tau(q+1, r+1)}-\frac{\tau(q+1, r+2) \tau(r+1)}{\tau(q+1, r+1) \tau(r+2)}
\end{aligned}
$$

Это равенство означает, что

$$
\frac{\tau(p+1) \tau(q+1, r+1)+\tau(q+1) \tau(p+1, r+1)}{\tau(r+1) \tau(p+1, q+1)}
$$

является периодической функцией от $r$ с периодом 1 и произвольной функцией от $p, q$. Поскольку никаких специальных свойств периодичности не предполагается, мы примем, что эта функция не зависит от $r$. Таким образом, мы приходим к соотношению

$$
\tau(p+1) \tau(q+1, r+1)+\tau(q+1) \tau(p+1, r+1)=h(2 p, 2 q) \tau(r+1) \tau(p+1, q+1),
$$

где $h$ может быть произвольной функцией от $p$ и $q$. В исходных переменных это уравнение имеет вид

$$
T(a+1) T(a-1)+T(s+1) T(s-1)=h(u-s-a, u+s+a) T(u+1) T(u-1) .
$$

Из граничных условий (7) при $a=0$ или $s=0$ следует, что $h=1$, и мы получаем уравнение Хироты (9). Заметим, что параметр $z$, входящий в линейные задачи, исчезает из нелинейного уравнения. На самом деле, это было ясно с самого начала, так как $z$ можно устранить из (32) преобразованием $\psi \rightarrow z^{p+q+r} \psi$. Тем не менее мы удерживаем этот параметр, поскольку он будет важен в дальнейшем.

Преимущество "киральных" переменных в том, что они разделяются в линейных задачах: первая задача не затрагивает $q$, а вторая не затрагивает $p$. Однако в отличие от "лабораторных" переменных $a, s, u$ "киральные" переменные не имеют непосредственного физического смысла. Переходя обратно к "лабораторным" переменным, введем функцию $\psi(p, q, r)=\Psi(q+r,-p-r, p+q)$ и перепишем линейные 
задачи (32) в виде

$$
\begin{aligned}
& \Psi(a, s, u)+z \frac{T(a-1, s+1, u) T(a, s-1, u+1)}{T(a, s, u) T(a-1, s, u+1)} \Psi(a-1, s+1, u)=\Psi(a-1, s, u+1), \\
& \Psi(a, s, u)-z \frac{T(a-1, s+1, u) T(a+1, s, u+1)}{T(a, s, u) T(a, s+1, u+1)} \Psi(a-1, s+1, u)=\Psi(a, s+1, u+1) .
\end{aligned}
$$

Вообще говоря, совместность линейных задач подразумевает наличие непрерывного семейства общих решений. Как мы видели, структура коэффициентных функций в нашем случае такова, что совместность эквивалентна наличию по крайней мере одного общего решения (см. работу [22], где этот факт был отмечен в другом контексте).

Ввиду того что $T$-функции могут тождественно обращаться в нуль при некоторых $(a, s)$, мы избавимся от знаменателей, перейдя к новой вспомогательной функции $F=T \Psi$, в терминах которой имеем

$$
\begin{aligned}
& T(a-1, s, u+1) F(a, s, u)+z T(a, s-1, u+1) F(a-1, s+1, u)= \\
& \quad=T(a, s, u) F(a-1, s, u+1), \\
& T(a, s+1, u+1) F(a, s, u)-z T(a+1, s, u+1) F(a-1, s+1, u)= \\
& \quad=T(a, s, u) F(a, s+1, u+1) .
\end{aligned}
$$

Отметим, что второе уравнение получается из первого с помощью преобразования $T(a, s, u) \rightarrow(-1)^{a s} T(-s,-a, u)$ (и такого же преобразования $F$ ), которое не меняет вид уравнения Хироты. Однако уравнение Хироты, записанное для функции $\widetilde{T}(a, s, u)=(-1)^{\left(a^{2}+s^{2}\right) / 2} T(a, s, u)$, сохраняет свой вид при преобразованиях из большей группы симметрии, состоящей из всех перестановок и изменений знаков переменных $a, s, u$, чего нельзя сказать о системе линейных задач (34). На самом деле, эта симметрия реализуется неявным образом. Чтобы сделать ее явной, запишем пару уравнений (34) в матричной форме

$$
\begin{aligned}
& \left(\begin{array}{cc}
T(a-1, s, u) & z T(a, s-1, u) \\
T(a, s+1, u) & -z T(a+1, s, u)
\end{array}\right)\left(\begin{array}{c}
F(a, s, u-1) \\
F(a-1, s+1, u-1)
\end{array}\right)= \\
& \quad=T(a, s, u-1)\left(\begin{array}{c}
F(a-1, s, u) \\
F(a, s+1, u)
\end{array}\right)
\end{aligned}
$$

и умножим обе части на матрицу, обратную матрице в левой части равенства. Воспользовавшись $T T$-соотношением, получим другую пару линейных задач:

$$
\begin{aligned}
& T(a+1, s+1, u) F(a, s, u)-z T(a+1, s, u+1) F(a, s+1, u-1)= \\
& \quad=T(a, s, u) F(a+1, s+1, u), \\
& T(a, s, u+1) F(a, s, u-1)-T(a, s-1, u) F(a, s+1, u)= \\
& \quad=T(a+1, s, u) F(a-1, s, u),
\end{aligned}
$$

которая эквивалентна паре (34) (и, значит, совместна с ней) по построению. Набор из четырех линейных задач (34), (36) обладает требуемой симметрией. Уравнение Хироты может быть выведено как условие совместности любых двух линейных 
задач из этих четырех, при этом два оставшихся уравнения удовлетворятся автоматически. Эти четыре линейных уравнения можно объединить в одно матричное уравнение

$$
\mathbb{T}(a, s, u)\left(\begin{array}{c}
F(a-1, s, u) \\
F(a, s+1, u) \\
F(a, s, u-1) \\
z F(a-1, s+1, u-1)
\end{array}\right)=0
$$

где $\mathbb{T}(a, s, u)$ - антисимметричная матрица,

$$
\mathbb{T}(a, s, u)=\left(\begin{array}{cccc}
0 & T(a, s, u-1) & -T(a, s+1, u) & T(a+1, s, u) \\
-T(a, s, u-1) & 0 & T(a-1, s, u) & T(a, s-1, u) \\
T(a, s+1, u) & -T(a-1, s, u) & 0 & -T(a, s, u+1) \\
-T(a+1, s, u) & -T(a, s-1, u) & T(a, s, u+1) & 0
\end{array}\right) .
$$

Из уравнения Хироты следует, что ее детерминант обращается в нуль и ранг равен 2. Симметричная форма записи линейных задач для уравнения Хироты была предложена в [23]. Более подробное обсуждение линейных задач и их симметрий можно найти в [19], [21], [23], [24].

\section{6. ПРЕОБРАЗОВАНИЯ БЕКЛУНДА}

Между функциями $T(a, s, u)$ и $F(a, s, u)$ имеется замечательное соотношение дуальности [1], [21]: их можно поменять ролями и понимать уравнения (34) как переопределенную систему линейных задач для функции $T$ с коэффициентами $F$. Требование совместности этих задач дает то же уравнение Хироты для $F$ :

$$
F(a, s, u+1) F(a, s, u-1)=F(a, s+1, u) F(a, s-1, u)+F(a+1, s, u) F(a-1, s, u) .
$$

Таким образом, любое решение линейных задач (34), где $T$-функции удовлетворяют уравнению Хироты, дает (авто)преобразование Беклунда, т.е. преобразование, переводящее решение нелинейного интегрируемого уравнения в другое решение того же уравнения.

Перепишем линейные задачи (34), изменив порядок членов и произведя сдвиги переменных:

$$
\begin{aligned}
& T(a+1, s, u) F(a, s, u+1)-T(a, s, u+1) F(a+1, s, u)= \\
& \quad=z T(a+1, s-1, u+1) F(a, s+1, u), \\
& T(a, s+1, u+1) F(a, s, u)-T(a, s, u) F(a, s+1, u+1)= \\
& \quad=z T(a+1, s, u+1) F(a-1, s+1, u) .
\end{aligned}
$$

Эти уравнения изображены графически на $(a, s)$-решетке на рис. 2. Они представляют собой преобразование Беклунда $T \rightarrow F$ в билинейной форме [8], [21]. Если задано семейство полиномов $T(a, s, u)$, удовлетворяющих уравнению Хироты, то можно поставить задачу о поиске полиномиальных решений уравнений (40). Легко видеть, что эти уравнения не совместны с граничными условиями для $F(a, s, u)$ и $T(a, s, u)$ типа "жирного крюка" с одинаковыми $K$ и $M$. Действительно, применив эти уравнения в угловой точке внутренней границы, видим, что если $K, M$ для $T$ и $F$ одинаковы, то граничные значения должны тождественно обращаться в нуль. 

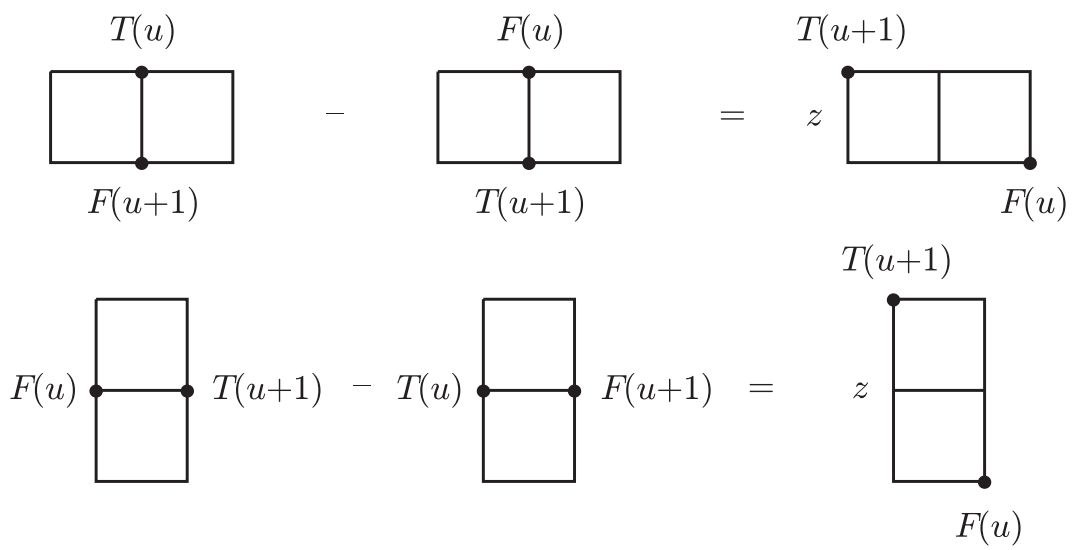

Рис. 2. Графическое представление уравнений (40) на $(a, s)$-решетке. Координаты $a$ и $s$ отложены соответственно по вертикальной и горизонтальной осям.

Однако можно непосредственно проверить, что уравнения (40) совместны с граничными условиями следующих двух типов:

$$
\begin{gathered}
F(a, s, u)=0, \text { если } \\
a<0, \text { или } a>0 \text { и } s<0, \text { или } a>K-1 \text { и } s>M \text {, }
\end{gathered}
$$

или

$$
F(a, s, u)=0, \quad \text { если }
$$

$$
a<0, \text { или } a>0 \quad \text { и } s<0 \text {, или } a>K \quad \text { и } s>M+1 .
$$

Это вновь условия типа "жирного крюка", но со сдвигом $K \rightarrow K-1$ или $M \rightarrow M+1$. Мы обозначим соответствующие преобразования через $\mathrm{BT}_{1}^{-}$и $\mathrm{BT}_{2}^{+}: F(a, s, u)=$ $\mathrm{BT}_{1}^{-}(T(a, s, u))$ для $(41)$ и $F(a, s, u)=\mathrm{BT}_{2}^{+}(T(a, s, u))$ для (42). Эти преобразования зависят от параметра $z$. Обратные преобразования $\mathrm{BT}_{1}^{+}=\left(\mathrm{BT}_{1}^{-}\right)^{-1}$ и $\mathrm{BT}_{2}^{-}=$ $\left(\mathrm{BT}_{2}^{+}\right)^{-1}$ определяются теми же формулами (40), если понимать их как линейные уравнения на $T$ при данных $F$. Более явным образом преобразования $\mathrm{BT}_{1}^{ \pm}, \mathrm{BT}_{2}^{ \pm}$ задаются формулами (47), (48), которые приведены ниже.

Повторив эти преобразования несколько раз, мы приходим к иерархии функций $T_{k, m}(a, s, u)(k=0,1, \ldots, K, m=0,1, \ldots, M)$ таких, что:

а) они удовлетворяют уравнению Хироты по $a, s, u$ при всех $k, m$;

б) они связаны преобразованиями Беклунда

$$
\begin{aligned}
& T_{k-1, m}(a, s, u)=\mathrm{BT}_{1}^{-}\left(T_{k, m}(a, s, u)\right), \\
& T_{k, m-1}(a, s, u)=\mathrm{BT}_{2}^{-}\left(T_{k, m}(a, s, u)\right) ;
\end{aligned}
$$

в) при $k=K, m=M$ имеет место соотношение

$$
T_{K, M}(a, s, u)=T(a, s, u) .
$$

Для неприводимых полиномиальных решений этот список нужно дополнить следующим "начальным условием": $T_{0,0}(a, s, u)=1$ при $a=0$ или при $s=0, a>0$, $T_{0,0}(a, s, u)=0$ в других случаях. Уровни иерархии нумеруются парой чисел $(k, m)$. 
Наинизший уровень - это $(0,0)$, а наивысший - $(K, M)$. Мы будем говорить, что $T$-функции $T_{k, m}(a, s, u)$ принадлежат уровню $(k, m)$.

Важно отметить, что параметр $z$, участвующий в определении преобразований Беклунда, может быть различным как для введенных выше преобразований двух разных типов, так и для последовательных преобразований одного и того же типа. Мы положим этот параметр равным собственному значению матрицы $g: z=x_{k}$ для перехода $(k, m) \rightarrow(k-1, m)$ и $z=y_{m}$ для $(k, m) \rightarrow(k, m-1)$. Таким образом, преобразование $\mathrm{BT}_{1}^{-}$запишется в виде

$$
\begin{aligned}
& T_{k, m}(a+1, s, u) T_{k-1, m}(a, s, u+1)-T_{k, m}(a, s, u+1) T_{k-1, m}(a+1, s, u)= \\
& \quad=x_{k} T_{k, m}(a+1, s-1, u+1) T_{k-1, m}(a, s+1, u), \\
& \quad T_{k, m}(a, s+1, u+1) T_{k-1, m}(a, s, u)-T_{k, m}(a, s, u) T_{k-1, m}(a, s+1, u+1)= \\
& \quad=x_{k} T_{k, m}(a+1, s, u+1) T_{k-1, m}(a-1, s+1, u),
\end{aligned}
$$

а преобразование $\mathrm{BT}_{2}^{-}$- в виде

$$
\begin{aligned}
& T_{k, m-1}(a+1, s, u) T_{k, m}(a, s, u+1)-T_{k, m-1}(a, s, u+1) T_{k, m}(a+1, s, u)= \\
& \quad=y_{m} T_{k, m-1}(a+1, s-1, u+1) T_{k, m}(a, s+1, u), \\
& T_{k, m-1}(a, s+1, u+1) T_{k, m}(a, s, u)-T_{k, m-1}(a, s, u) T_{k, m}(a, s+1, u+1)= \\
& \quad=y_{m} T_{k, m-1}(a+1, s, u+1) T_{k, m}(a-1, s+1, u)
\end{aligned}
$$

$(k=1, \ldots, K, m=1, \ldots, M)$. Если не обращать внимание на зависимость от $u$ (которая исчезает в пределе $u \rightarrow \infty$ ), можно рассматривать эти формулы как билинейные соотношения между характерами, упомянутые в разделе 3 (см. уравнения (22) и комментарии после них). Нетрудно заметить, что каждое из уравнений в $(44),(45)$ в действительности является динамическим уравнением на функцию от трех переменных, а не от пяти. Например, первое уравнение в (44) действует в подпространствах $m=$ const и $u+s+a=$ const. После ограничения на соответствующие трехмерные гиперплоскости в линейном пространстве с координатами $a, s, u, k, m$ каждое из этих уравнений линейной заменой переменных может быть приведено к стандартному виду уравнения Хироты.

Для полноты рассуждений дадим симметричное описание преобразований Беклунда с помощью матричных уравнений вида (37). Для прямого и обратного преобразований введем две антисимметричные $(4 \times 4)$-матрицы $\mathbb{T}^{( \pm 1)}(a, s, u)$ типа $(38)$ :

$$
\mathbb{T}^{(\varepsilon)}(a, s, u)=\left(\begin{array}{cccc}
0 & T(a, s, u-\varepsilon) & -T(a, s+\varepsilon, u) & T(a+\varepsilon, s, u) \\
-T(a, s, u-\varepsilon) & 0 & T(a-\varepsilon, s, u) & T(a, s-\varepsilon, u) \\
T(a, s+\varepsilon, u) & -T(a-\varepsilon, s, u) & 0 & -T(a, s, u+\varepsilon) \\
-T(a+\varepsilon, s, u) & -T(a, s-\varepsilon, u) & T(a, s, u+\varepsilon) & 0
\end{array}\right),
$$

где $\varepsilon= \pm 1$. Тогда преобразования $T_{k-1, m}=\mathrm{BT}_{1}^{-}\left(T_{k, m}\right), T_{k, m+1}=\mathrm{BT}_{2}^{+}\left(T_{k, m}\right)$ задаются матричным уравнением

$$
\mathbb{T}_{k, m}^{(+1)}(a, s, u)\left(\begin{array}{cc}
T_{k-1, m}(a-1, s, u) & T_{k, m+1}(a-1, s, u) \\
T_{k-1, m}(a, s+1, u) & T_{k, m+1}(a, s+1, u) \\
T_{k-1, m}(a, s, u-1) & T_{k, m+1}(a, s, u-1) \\
x_{k} T_{k-1, m}(a-1, s+1, u-1) & y_{m+1} T_{k, m+1}(a-1, s+1, u-1)
\end{array}\right)=0,
$$


а обратные преобразования $T_{k+1, m}=\mathrm{BT}_{1}^{+}\left(T_{k, m}\right), T_{k, m-1}=\mathrm{BT}_{2}^{-}\left(T_{k, m}\right)-$ уравнением

$$
\mathbb{T}_{k, m}^{(-1)}(a, s, u)\left(\begin{array}{cc}
T_{k+1, m}(a+1, s, u) & T_{k, m-1}(a+1, s, u) \\
T_{k+1, m}(a, s-1, u) & T_{k, m-1}(a, s-1, u) \\
T_{k+1, m}(a, s, u+1) & T_{k, m-1}(a, s, u+1) \\
x_{k+1} T_{k+1, m}(a+1, s-1, u+1) & y_{m} T_{k, m-1}(a+1, s-1, u+1)
\end{array}\right)=0,
$$

где $\mathbb{T}_{k, m}^{( \pm)}(a, s, u)$ - матрицы (46) с матричными элементами на уровне $(k, m)$.

Аккуратный анализ уравнений (44)-(48) показывает, что граничные значения $T$-функций на каждом уровне $(k, m)$ подчиняются тем же соотношениям, что и на высшем уровне. На внешних границах $T$-функции имеют специальный вид, подобный $(7)$, т.е. $T_{k, m}(0, s, u)$ - это некоторая функция от $u-s$, a $T_{k, m}(a, 0, u)$ - та же функция от $u+a$. Введем для этой функции специальное обозначение:

$$
T_{k, m}(0, s, u)=Q_{k, m}(u-s), \quad T_{k, m}(a, 0, u)=Q_{k, m}(u+a) .
$$

Полиномы $Q_{k, m}(u)$ играют очень важную роль. Мы отождествим их с собственными значениями $Q$-операторов Бакстера. Полином $Q_{K, M}(u)=\phi(u)$ является фиксированным внешним данным, определяющим модель. Полиномы $Q_{k, m}(u)$ на более низких уровнях должны быть найдены в процессе решения. По аналогии с (8) зафиксируем их старшие коэффициенты, положив их равными 1. Применив уравнения (44), (45) на внутренних границах, можно убедиться, что если (28) выполняется на высшем уровне, то T-функции на внутренних границах, принадлежащие каждому из уровней $(k, m)$, связаны аналогичным соотношением

$$
T_{k, m}(k, m+n, u)=(-1)^{n m}\left(\operatorname{sdet}_{k, m}\right)^{n} T_{k, m}(k+n, m, u), \quad n \geqslant 0,
$$

где матрица $g_{k, m} \in G L(k \mid m)$ определена в $(23)$ и $\operatorname{sdet} g_{k, m}=x_{1} \ldots x_{k} /\left(y_{1} \ldots y_{m}\right)$.

Теперь мы видим, что применение преобразований $\mathrm{BT}_{1}^{-}$и $\mathrm{BT}_{2}^{-}$к решению уравнения Хироты в области $\mathrm{H}(K \mid M)$ с граничными условиями (27) позволяет последовательно преобразовать его в тривиальное решение в вырожденной области $\mathrm{H}(0 \mid 0)$. Как будет показано ниже, с помощью этой “процедуры раздевания" можно строить решения исходной задачи.

\section{7. РЕКУРРЕНТНЫЕ СООТНОШЕНИЯ ДЛЯ ОПЕРАТОРНОГО ПРОИЗВОДЯЩЕГО РЯДА}

Преобразования Беклунда из предыдущего раздела можно переформулировать в операторном виде как рекуррентные соотношения для некоторого разностного оператора бесконечного порядка. Рассмотрим следующий оператор:

$$
\mathcal{W}(u)=\sum_{s \geqslant 0} \frac{T(1, s, u+s-1)}{\phi(u)} e^{2 s \partial_{u}}
$$

(общий знаменатель введен для нормировки). Он служит некоммутативным производящим рядом для $T$-функций $T(1, s, u)$. Аналогичные объекты, представляющие собой производящие ряды для функций $T_{k, m}(1, s, u)$, можно ввести на любом уровне $(k, m)$ :

$$
\mathcal{W}_{k, m}(u)=\sum_{s \geqslant 0} \frac{T_{k, m}(1, s, u+s-1)}{Q_{k, m}(u)} e^{2 s \partial_{u}}
$$


Ясно, что $\mathcal{W}_{0,0}(u)=1$ (вспомним, что $T_{0,0}(1, s, u)=0$ при $s \neq 0$, а $T_{0,0}(1,0, u)=$ $\left.Q_{0,0}(u+1)=1\right)$. Отметим также, что ряд, формально обратный к $(52)$, порождает T-функции $T_{k, m}(a, 1, u)$ :

$$
\mathcal{W}_{k, m}^{-1}(u)=\sum_{a \geqslant 0}(-1)^{a} e^{2 a \partial_{u}} \frac{T_{k, m}(a, 1, u-a-1)}{Q_{k, m}(u-2)}
$$

(доказательство этого факта см. в работе [9]).

Пусть

$$
\begin{aligned}
X_{k, m}(u) & =x_{k} \frac{Q_{k, m}(u+2) Q_{k-1, m}(u-2)}{Q_{k, m}(u) Q_{k-1, m}(u)}, \\
Y_{k, m}(u) & =y_{m} \frac{Q_{k, m-1}(u+2) Q_{k, m}(u-2)}{Q_{k, m-1}(u) Q_{k, m}(u)} .
\end{aligned}
$$

Пользуясь линейными задачами при $a=0$, прямым вычислением можно доказать следующие рекуррентные соотношения для операторов $\mathcal{W}_{k, m}(u)$ :

$$
\begin{aligned}
& \mathcal{W}_{k-1, m}(u)=\left(1-X_{k, m}(u) e^{2 \partial_{u}}\right) \mathcal{W}_{k, m}(u), \\
& \mathcal{W}_{k, m+1}(u)=\left(1-Y_{k, m+1}(u) e^{2 \partial_{u}}\right) \mathcal{W}_{k, m}(u) .
\end{aligned}
$$

Эти формулы являются операторными (и зависящими от $u$ ) аналогами равенств (26). Оператор сдвига $\mathbf{t}=e^{2 \partial_{u}}$ играет роль переменной $t$, а переменная $u$ в (26) отсутствует. Заметим также, что $X_{k, m}(u), Y_{k, m}(u)$ в пределе $u \rightarrow \infty$ переходят в $x_{k}, y_{m}$.

Здесь мы приведем некоторые детали доказательства соотношений (56) (см. также [9], где доказана несколько иная версия этих рекуррентных соотношений). Рассмотрим первое соотношение. Имеем

$$
\mathcal{W}_{k-1, m}(u)-\mathcal{W}_{k, m}(u)=\sum_{s \geqslant 0}\left[\frac{T_{k-1, m}(1, s, u+s-1)}{Q_{k-1, m}(u)}-\frac{T_{k, m}(1, s, u+s-1)}{Q_{k, m}(u)}\right] e^{2 s \partial_{u}} .
$$

Для того чтобы преобразовать выражение в квадратных скобках, напишем первое уравнение в (44) при $a=0$ в виде

$$
\begin{aligned}
& \frac{T_{k, m}(1, s, u+s-1)}{Q_{k, m}(u)}-\frac{T_{k-1, m}(1, s, u+s-1)}{Q_{k-1, m}(u)}= \\
& =x_{k} \frac{Q_{k, m}(u+2) Q_{k-1, m}(u-2)}{Q_{k, m}(u) Q_{k-1, m}(u)} \frac{T_{k, m}(1, s-1, u+s)}{Q_{k, m}(u+2)}
\end{aligned}
$$

и продолжим равенство (57):

$$
\mathcal{W}_{k-1, m}(u)-\mathcal{W}_{k, m}(u)=-X_{k, m}(u) \sum_{s \geqslant 0} \frac{T_{k, m}(1, s-1, u+s)}{Q_{k, m}(u+2)} e^{2 s \partial_{u}} .
$$

В силу того что $T_{k, m}(1,-1, u)=0$, сумму в правой части можно записать в виде

$$
\sum_{s \geqslant 0} \frac{T_{k, m}(1, s-1, u+s)}{Q_{k, m}(u+2)} e^{2 s \partial_{u}}=e^{2 \partial_{u}} \sum_{s \geqslant 0} \frac{T_{k, m}(1, s, u+s-1)}{Q_{k, m}(u)}=e^{2 \partial_{u}} \mathcal{W}_{k, m}(u),
$$

и первое рекуррентное соотношение доказано. Второе доказывается совершенно аналогично.

\section{8. ФОРМУЛЫ ФАКТОРИЗАЦИИ И ТQ-СООТНОШЕНИЯ}

Рекуррентные соотношения, установленные в предыдущем разделе, позволяют представить операторный производящий ряд (51) в замкнутой факторизованной 
форме, где каждый сомножитель содержит только $Q$-функции. Точнее, $\mathcal{W}_{K, M}(u)$ можно получить как результат последовательного применения рекуррентных соотношений $(56) \mathrm{k} \mathcal{W}_{0,0}(u)=1$. Действуя таким образом и двигаясь сначала в $m$-направлении из $(0,0)$ в $(0, M)$, а затем в $k$-направлении из $(0, M)$ в $(K, M)$, получаем

$$
\mathcal{W}_{K, M}(u)=\prod_{K \geqslant k \geqslant 1}^{\leftarrow}\left(1-X_{k, M}(u) e^{2 \partial_{u}}\right)^{-1} \cdot \prod_{M \geqslant m \geqslant 1}^{\leftarrow}\left(1-Y_{0, m}(u) e^{2 \partial_{u}}\right)
$$

где упорядоченное произведение определено как

$$
\prod_{J \geqslant i \geqslant I}^{\leftarrow} A_{i}=A_{J} A_{J-1} \ldots A_{I+1} A_{I}
$$

Применив рекуррентные соотношения в другом порядке (двигаясь сначала в $k$-направлении, а затем в $m$-направлении), приходим к другому по форме, но эквивалентному представлению

$$
\mathcal{W}_{K, M}(u)=\prod_{M \geqslant m \geqslant 1}^{\leftarrow}\left(1-Y_{K, m}(u) e^{2 \partial_{u}}\right) \cdot \prod_{K \geqslant k \geqslant 1}^{\leftarrow}\left(1-X_{k, 0}(u) e^{2 \partial_{u}}\right)^{-1}
$$

На самом деле, можно применять соотношения (56) в любом другом порядке, который определяется выбранным путем из точки $(0,0)$ в точку $(K, M)$. Каждый шаг $(k, m) \rightarrow(k+1, m)$ в $k$-направлении добавляет множитель $\left(1-X_{k+1, m}(u) e^{2 \partial_{u}}\right)^{-1}$, а каждый шаг $(k, m) \rightarrow(k, m+1)$ в $m$-направлении - множитель $\left(1-Y_{k, m+1}(u) e^{2 \partial_{u}}\right)$; эти множители нужно перемножить в соответствии с порядком шагов. Это дает много других способов факторизации оператора $\mathcal{W}_{K, M}(u)$. Их эквивалентность следует из условия совместности рекуррентных соотношений (56), которое выражается дискретным условием "нулевой кривизны"

$$
\left(1-Y_{k-1, m+1}(u) e^{2 \partial_{u}}\right)\left(1-X_{k, m}(u) e^{2 \partial_{u}}\right)=\left(1-X_{k, m+1}(u) e^{2 \partial_{u}}\right)\left(1-Y_{k, m+1}(u) e^{2 \partial_{u}}\right)
$$

на $(k, m)$-решетке. Две стороны этого равенства соответствуют двум различным способам получить $\mathcal{W}_{k-1, m+1}(u)$ из $\mathcal{W}_{k, m}(u)$.

Равенства (59) и (60), а также аналогичные равенства с другим упорядочением представляют собой обобщенные $T Q$-соотношения Бакстера в производящей форме. Приравняв коэффициенты при различных степенях оператора $e^{2 \partial_{u}}$, получим выражения для $T$-функций $T(1, s, u)=T_{K, M}(1, s, u)$ через $X_{k, m}, Y_{k, m}$, т.е. через $Q$-функции $Q_{k, m}(u)$ с $1 \leqslant k \leqslant K, 1 \leqslant m \leqslant M$. Например, простейшее $T Q$-соотношение, содержащееся в (59), имеет вид

$$
\frac{T_{K, M}(1,1, u)}{Q_{K, M}(u)}=\sum_{k=1}^{K} X_{k, M}(u)-\sum_{m=1}^{M} Y_{0, m}(u),
$$

где $X_{k, M}(u), Y_{0, m}(u)$ должны быть выражены через $Q$-функции в соответствии с (54), (55). Нули последних должны удовлетворять системе уравнений Бете, которые выводятся в следующем разделе.

\section{QQ-СООТНОШЕНИЕ И УРАВНЕНИЯ БЕТЕ}

Наша отправная точка в этом разделе - дискретное условие нулевой кривизны (61). Сравнив коэффициенты при различных степенях оператора сдвига, мы 
замечаем, что равенство

$$
Y_{k-1, m+1}(u) X_{k, m}(u+2)=X_{k, m+1}(u) Y_{k, m+1}(u+2)
$$

выполняется тождественно, и, таким образом, получаем единственное нетривиальное соотношение

$$
Y_{k-1, m+1}(u)+X_{k, m}(u)=X_{k, m+1}(u)+Y_{k, m+1}(u),
$$

которое после подстановок (54), (55) превращается в функциональное уравнение для $Q$-функций. Как показывает простое вычисление, оно эквивалентно следующему билинейному уравнению (“Q $Q$-соотношению" из работы $[9])$ :

$$
\begin{gathered}
x_{k} Q_{k-1, m-1}(u) Q_{k, m}(u+2)-y_{m} Q_{k, m}(u) Q_{k-1, m-1}(u+2)= \\
=\left(x_{k}-y_{m}\right) Q_{k-1, m}(u) Q_{k, m-1}(u+2)
\end{gathered}
$$

для полиномиальных функций

$$
Q_{k, m}(u)=\prod_{j=1}^{N_{k, m}}\left(u-u_{j}^{(k, m)}\right) .
$$

Заметим, что $Q Q$-соотношение (63) может быть приведено к билинейному разностному уравнению Хироты стандартного вида для "киральных" переменных $u$, $m,-k$ путем перехода к функции

$$
\mathcal{Q}_{k, m}(u)=a_{k, m} e^{\left(\beta_{k}+\gamma_{m}\right) u} Q_{k, m}(u),
$$

где вновь введенные параметры $\beta_{k}$ и $\gamma_{m}$ связаны с $x_{k}, y_{m}$ формулами

$$
x_{k}=e^{2\left(\beta_{k}-\beta_{k-1}\right)}, \quad y_{m}=e^{2\left(\gamma_{m-1}-\gamma_{m}\right)} .
$$

Они фиксируются однозначно, если положить $\beta_{0}=\gamma_{0}=0$. В результате преобразования (65) коэффициенты $x_{k}, y_{m}$ в (63) (а также в $\left.(54),(55)\right)$ исчезают, и при подходящем выборе $a_{k, m} Q Q$-соотношение принимает бескоэффициентную форму

$$
\mathcal{Q}_{k-1, m-1}(u) \mathcal{Q}_{k, m}(u+2)-\mathcal{Q}_{k, m}(u) \mathcal{Q}_{k-1, m-1}(u+2)=\mathcal{Q}_{k-1, m}(u) \mathcal{Q}_{k, m-1}(u+2),
$$

предложенную в [9].

$Q Q$-соотношение (63) дает наиболее простой и прозрачный способ вывода уравнений Бете для корней полиномов $Q_{k, m}(u)$. Последовательно положив $u$ в (63) равным корням всех $Q$-функций, входящих в уравнение, мы получаем набор соотношений, которые после некоторых преобразований могут быть приведены к виду

$$
\begin{aligned}
\frac{Q_{k-1, m}\left(u_{j}^{(k, m)}\right) Q_{k, m}\left(u_{j}^{(k, m)}-2\right) Q_{k+1, m}\left(u_{j}^{(k, m)}+2\right)}{Q_{k-1, m}\left(u_{j}^{(k, m)}-2\right) Q_{k, m}\left(u_{j}^{(k, m)}+2\right) Q_{k+1, m}\left(u_{j}^{(k, m)}\right)} & =-\frac{x_{k}}{x_{k+1}}, \\
\frac{Q_{k, m+1}\left(u_{j}^{(k, m)}\right) Q_{k, m}\left(u_{j}^{(k, m)}-2\right) Q_{k, m-1}\left(u_{j}^{(k, m)}+2\right)}{Q_{k, m+1}\left(u_{j}^{(k, m)}-2\right) Q_{k, m}\left(u_{j}^{(k, m)}+2\right) Q_{k, m-1}\left(u_{j}^{(k, m)}\right)} & =-\frac{y_{m+1}}{y_{m}}, \\
\frac{Q_{k+1, m}\left(u_{j}^{(k, m)}\right) Q_{k, m-1}\left(u_{j}^{(k, m)}+2\right)}{Q_{k+1, m}\left(u_{j}^{(k, m)}+2\right) Q_{k, m-1}\left(u_{j}^{(k, m)}\right)} & =\frac{x_{k+1}}{y_{m}}, \\
\frac{Q_{k, m+1}\left(u_{j}^{(k, m)}\right) Q_{k-1, m}\left(u_{j}^{(k, m)}-2\right)}{Q_{k, m+1}\left(u_{j}^{(k, m)}-2\right) Q_{k-1, m}\left(u_{j}^{(k, m)}\right)} & =\frac{y_{m+1}}{x_{k}} .
\end{aligned}
$$


Эти равенства выполняются внутри прямоугольника $[0, K] \times[0, M]$ на $(k, m)$-решетке и служат элементарными строительными блоками для системы уравнений Бете. Каждая такая система соответствует зигзагообразному "раздевающему" пути из точки $(K, M)$ в точку $(0,0)$. На отрезках такого пути $(k+1, m) \rightarrow(k, m) \rightarrow(k-1, m)$, $(k, m+1) \rightarrow(k, m) \rightarrow(k, m-1),(k+1, m) \rightarrow(k, m) \rightarrow(k, m-1)$ и $(k, m+1) \rightarrow(k, m) \rightarrow$ $(k-1, m)$ надо использовать соответственно уравнения $(68),(69),(70)$ и (71). Эти системы различны по виду, но эквивалентны между собой. Более подробное обсуждение этого вопроса имеется в [9]. В качестве примера приведем здесь цепочку уравнений Бете для простейшего пути $(K, M) \longrightarrow(0, M) \longrightarrow(0,0)$. При движении из $(K, M)$ в $(0, M)$ имеем уравнения

$$
\frac{Q_{k-1, M}\left(u_{j}^{(k, M)}\right) Q_{k, M}\left(u_{j}^{(k, M)}-2\right) Q_{k+1, M}\left(u_{j}^{(k, M)}+2\right)}{Q_{k-1, M}\left(u_{j}^{(k, M)}-2\right) Q_{k, M}\left(u_{j}^{(k, M)}+2\right) Q_{k+1, M}\left(u_{j}^{(k, M)}\right)}=-\frac{x_{k}}{x_{k+1}}
$$

$(k=1, \ldots, K-1)$. Они находятся в согласии с цепочкой уравнений Бете, написанной в [25] для бозонного случая. В точке поворота уравнение имеет вид

$$
\frac{Q_{1, M}\left(u_{j}^{(0, M)}\right) Q_{0, M-1}\left(u_{j}^{(0, M)}+2\right)}{Q_{1, M}\left(u_{j}^{(0, M)}+2\right) Q_{0, M-1}\left(u_{j}^{(0, M)}\right)}=\frac{x_{1}}{y_{M}} .
$$

Наконец, при движении из $(0, M)$ в $(0,0)$ имеем уравнения

$$
\frac{Q_{0, m+1}\left(u_{j}^{(0, m)}\right) Q_{0, m}\left(u_{j}^{(0, m)}-2\right) Q_{0, m-1}\left(u_{j}^{(0, m)}+2\right)}{Q_{0, m+1}\left(u_{j}^{(0, m)}-2\right) Q_{0, m}\left(u_{j}^{(0, m)}+2\right) Q_{0, m-1}\left(u_{j}^{(0, m)}\right)}=-\frac{y_{m+1}}{y_{m}}
$$

$(m=1,2, \ldots, M-1)$.

\section{0. ЗАКЛЮЧЕНИЕ}

Мы получили решение $T T$-соотношения (уравнения Хироты), удовлетворяющее всем требуемым граничным условиям и условиям аналитичности. Это решение дается детерминантной формулой (10), где полиномы $T(1, s, u)$ находятся из разложения (51) факторизованного оператора (59). Коэффициенты разложения выражаются через $Q$-функции посредством уравнений (54), (55), причем корни полиномов $Q_{k, m}(u)$ связаны системой уравнений Бете, которая выводится из билинейного $Q Q$-соотношения (63). Следует отметить, что это решение не единственно. При данных граничных условиях имеется конечное множество решений, соответствующих различным квантовым состояниям обобщенной спиновой цепочки.

Подчеркнем, что наше решение, типичное для квантовых задач, решаемых анзацем Бете, было получено чисто классическими методами теории солитонных уравнений на решетке. Ключевую роль играют преобразования Беклунда для разностного уравнения Хироты. С точки зрения теории классических солитонных уравнений наш метод заключается в построении цепочки последовательных преобразований Беклунда, которая сводит задачу к тривиальной. Каждое из этих преобразований имеет непрерывный параметр (классический спектральный параметр), который отождествляется с собственным значением матрицы, задающей твистованные граничные условия в квантовой интегрируемой модели. 
Благодарности. Автор благодарен В. Казакову и А. Сорину за многочисленные обсуждения и сотрудничество в [9]. Он выражает благодарность за гостеприимство организаторам Рабочего Совещания "Классические и квантовые интегрируемые системы" (Дубна, январь 2007), где была представлена часть результатов. Работа была частично поддержана РФФИ (грант № 06-01-92054-CE ${ }_{a}$ ), INTAS (грант № 03-51-6346), а также Программой поддержки ведущих научных школ (грант № НШ-8004.2006.2) и ANR GIMP (грант № ANR-05-BLAN-0029-01).

\section{Список литературы}

[1] I. Krichever, O. Lipan, P. Wiegmann, A. Zabrodin, Comm. Math. Phys., 188:2 (1997), 267-304; hep-th/9604080.

[2] A. Zabrodin, Internat. J. Modern Phys. B, 11 (1997), 3125-3158; hep-th/9610039.

[3] А. В. Забродин, ТМФ, 116 (1998), 54-100.

[4] V. Bazhanov, N. Reshetikhin, J. Phys. A, 23:9 (1990), 1477-1492.

[5] A. Klümper, P. Pearce, Physica A, 183 (1992), 304-350.

[6] A. Kuniba, T. Nakanishi, J. Suzuki, Internat. J. Modern Phys. A, 9 (1994), 5215-5266; hep-th/9309137.

[7] Z. Tsuboi, J. Phys. A, 30 (1997), 7975-7991.

[8] R. Hirota, J. Phys. Soc. Japan, 50:11 (1981), 3785-3791.

[9] V. Kazakov, A. Sorin, A. Zabrodin, Supersymmetric Bethe Ansatz and Baxter Equations from Discrete Hirota Dynamics, hep-th/0703147.

[10] П. П. Кулиш, Е. К. Склянин, Зап. науч. сем. ЛОМИ, 95 (1980), 129-160.

[11] П. П. Кулиш, Зап. науч. сем. ЛОМИ, 145 (1985), 140-163.

[12] V. Kac, Adv. Math., 26 (1977), 8-96; "Representations of classical Lie superalgebras", Differential geometrical methods in mathematical physics, II (Proc. Conf., Univ. Bonn, Bonn, 1977), Lecture Notes in Math., 676, Springer, New York, 1978, 597-626.

[13] A. Baha Balantekin, I. Bars, J. Math. Phys., 22:6 (1981), 1149-1162.

[14] I. Bars, B. Morel, H. Ruegg, J. Math. Phys., 24:9 (1983), 2253-2262.

[15] Л. А. Тахтаджян, Л. Д. Фаддеев, УМН, 34:5(209) (1979), 13-63.

[16] L. Faddeev, Internat. J. Modern Phys. A, 10:13 (1995), 1845-1878; hep-th/9404013.

[17] Н. М. Боголюбов, А. Г. Изергин, В. Е. Корепин, Коррелячионные функции интегрируемых систем и квантовый метод обратной задачи, Наука, М., 1992.

[18] E. Date, M. Jimbo, T. Miwa, J. Phys. Soc. Japan, 51:12 (1982), 4116-4124.

[19] А. В. Забродин, ТМФ, 113:2 (1997), 179-230.

[20] I. Macdonald, Symmetric Functions and Hall Polynomials, Oxford Univ. Press, New York-London, 1995.

[21] S. Saito, N. Saitoh, Phys. Lett. A, 120 (1987), 322-326; J. Math. Phys., 28:5 (1987), $1052-1055$.

[22] I. Krichever, Characterizing Jacobians via trisecants of the Kummer Variety, math. AG/0605625.

[23] N. Shinzawa, S. Saito, J. Phys. A, 31:19 (1998), 4533-4540; solv-int/9801002.

[24] N. Shinzawa, J. Phys. A, 33:21 (2000), 3957-3970; solv-int/9907016; N. Shinzawa, R. Hirota, J. Phys. A, 36:16 (2003), 4667-4675; The Bäcklund transformation equations for the ultradiscrete KP equation, nlin.SI/0212014.

[25] P. Kulish, N. Reshetikhin, J. Phys. A, 16 (1983), L591-L596. 